\title{
Correction
}

\section{Correction: Yaple et al., Transcranial Alternating Current Stimulation Modulates Risky Decision Making in a Frequency-Controlled Experiment (eNeuro November/December 2017, 4(6) e0136-17.2017 1-10 https://doi.org/10.1523/ENEURO.0136-17.2017)}

In the article "Transcranial Alternating Current Stimulation Modulates Risky Decision Making in a Frequency-Controlled Experiment" by Zachary Yaple, Mario Martinez-Saito, Matteo Feurra, Anna Shestakova, and Vasily Klucharev, which appeared as e0136-17.2017 in the November/December 2017 issue, 4(6), author Bhuvanesh Awasthi was inadvertently omitted from the author list. The authors apologize for the oversight. The corrected author list is as follows: Zachary Yaple, ${ }^{1}$ Mario MartinezSaito, ${ }^{1}$ Bhuvanesh Awasthi, ${ }^{1}$ Matteo Feurra, ${ }^{1,2}$ Anna Shestakova, ${ }^{1}$ and Vasily Klucharev ${ }^{1,2}$. Their affiliations are as follows: ${ }^{1}$ Centre for Cognition and Decision Making, National Research University Higher School of Economics, 20 Myasnitskaya Ulitsa, Moscow 109316, Russian Federation, ${ }^{2}$ School of Psychology, National Research University Higher School of Economics, Armyanskiy per.4,c2, Moscow 109316, Russian Federation. The Author Contributions footnote should have read "Author contributions: Z.Y., M.F., A.S., B.A., and V.K. designed research; Z.Y. performed research; Z.Y. and M.M. analyzed data; Z.Y. wrote the paper." This correction does not affect the conclusions of the paper. The 5th author has been added in the online PDF version. 